\title{
ON SYMMETRY IN PERIODIC SOLUTIONS OF HAMILTONIAN SYSTEMS
}

\author{
ARTHUR R. JONES
}

(Received 28 January 1965)

\section{Introduction}

In this paper the theory of periodic solutions of analytic Hamiltonian systems of differential equations, which is due to Cherry [5], is specialized to systems which have one symmetry property.

We shall consider conservative systems with 2 degrees of freedom

$$
\frac{d x_{k}}{d t}=\frac{\partial F}{\partial y_{k}}, \quad \frac{d y_{k}}{d t}=-\frac{\partial F}{\partial x_{k}} \quad(k=1,2)
$$

in which the Hamiltonian $F$ is an analytic function of the $x_{k}, y_{k}$. In vector notation such a system may be written as

$$
\frac{d x}{d t}=J F_{x}
$$

where $x$ is the vector with components $x_{1}, x_{2}, y_{1}, y_{2} ; J$ is the skew-symmetric matrix

$$
\left[\begin{array}{rc}
0 & I_{2} \\
-I_{2} & 0
\end{array}\right]
$$

( $I_{2}$ being the $2 \times 2$ unit matrix) and $F_{x}$ is the gradient of $F$. Later we shall change variables and shall then use $\zeta, z$ and $Z$ to denote vectors with components $\zeta_{1}, \zeta_{2}, \omega_{1}, \omega_{2} ; z_{1}, z_{2}, u_{1}, u_{2}$ and $Z_{1}, Z_{2}, U_{1}, U_{2}$ respectively.

In the usual dynamical interpretation of (1.1), $x_{1}$ and $x_{2}$ are the coordinates of a particle moving in a plane and any path which the particle describes in this plane is called an orbit of the system. $y_{1}$ and $y_{2}$ are the corresponding momenta and $F$ is the net energy of the particle.

Symmetries in the dynamical system are thus characterized by properties of $F$ - the energy function. If the dynamical system is reversible, for example, then the energy is the same when the momenta are reversed and so $F$ has the property that for all values of the $x_{k}, y_{k}$

$$
\begin{aligned}
& F\left(x_{1}, x_{2}, y_{1}, y_{2}\right)=F\left(x_{1}, x_{2},-y_{1},-y_{2}\right) \text {. } \\
& 463
\end{aligned}
$$


Symmetry of the dynamical system about the $x_{1}$-axis is characterized in a similar way by the identity

$$
F\left(x_{1}, x_{2}, y_{1}, y_{2}\right)=F\left(x_{1},-x_{2},-y_{1}, y_{2}\right) \text {. }
$$

We shall say, therefore, that the system (1.1) is symmetric if, in vector notation, $F$ satisfies the identity

$$
F(x)=F(V x)
$$

where $V$ is one of the following diagonal matrices:

$$
\pm \operatorname{diag}(1,1,-1,-1) \text { or } \pm \operatorname{diag}(1,-1,-1,1) \text {. }
$$

Concerning the solutions of a symmetric system (1.1) we note that if $x=\phi(t)$ is a solution then so is $x=V \phi(-t)$. The solutions of a symmetric system therefore occur in pairs. Each member of a pair may be regarded as the reflection, in the appropriate axis of symmetry, of the other member. Exceptionally two members of a pair may coincide to give a solution $x=\phi(t)$ which satisfies the identity

$$
\phi(t)=V \phi(-t) .
$$

Such a solution is its own reflection and we shall therefore say that it is symmetric. At $t=0$ the orbit of such a solution has a point of zero velocity or else an orthogonal crossing of one of the coordinate axes, according to the type of symmetry which $V$ represents.

A solution $x=\phi(t)$ is said to be periodic if $\phi(t)=\phi(t+T)$ for some $T>0$ and the smallest such $T$ is called the period of the solution. A periodic solution has a closed curve as its orbit and a symmetric periodic solution has an orbit with two distinct orthogonal crossings of the axis of symmetry, one at $t=0$ and the other at $t=T / 2$ : c.f. [2; vol I, p. 745].

The existence of periodic solutions near a symmetric periodic solution has been investigated by Birkhoff [2] in connection with the restricted 3body problem. More recently De Vogelaere [6], [7] has announced some interesting results for the Störmer problem. Cherry's methods lead to results which appear to be more detailed, however, and which can easily be extended to systems with more than 2 degrees of freedom.

Cherry defines a solution of (1.1) to be periodic if it can be expanded as a convergent Laurent series in $e^{\nu t}$ where, for a real period, $v$ is pure imaginary. A periodic solution is said to be regular if certain convergence criteria are satisfied [5; pp. 185-186]. Of the characteristic exponents of such a solution, two may be denoted by $\pm \lambda$ while the other two are zero (modulo $\nu$ ). $\lambda / \nu$ is called the characteristic ratio of the periodic solution and $\lambda$ may be determined so that it satisfies $-\frac{1}{2}<R 1 \lambda / v \leqq \frac{1}{2}$. 
Cherry considers the existence of periodic solutions near a given regular periodic solution which we shall call the generating solution and shall denote by

$$
x=\phi\left(e^{\nu t}\right) .
$$

Let $\lambda / v$ be its characteristic ratio. Cherry shows that if $\lambda / v$ is irrational then the generating solution can be embedded in an analytic family of periodic solutions called Family I. But if $\lambda / v$ is rational there are additional families of periodic solutions which contain the generating solution. These families are said to branch from Family I at the generating solution. The branching families give orbits which close after $\nu_{0}$ circuits of the orbits in Family I where $\lambda_{0} / v_{0}$ is the reduced form of the rational $\lambda / v$.

It is a fairly trivial result that if the system (1.1) and the generating solution (1.5) are symmetric then so are the members of Family $\mathrm{I}$.

Our main results for symmetric systems are as follows: When the generating solution is symmetric with $\lambda_{0} \neq 0$ then in general the branching families are composed of periodic solutions which are symmetric. When $\nu_{0} \geqq 4$ two branching families are obtained in general (Families II and III) but when $v_{0}=3$ or $v_{0}=2$ there is only one such family (Family II).

When $\lambda_{0}=0$, however, there are several different cases which are of general occurrence and in one of these cases we obtain branching families whose members are unsymmetric.

The diagrams $[5 ;$ p. 177] which summarize the reality and stability properties of the branching families when $\lambda_{0} \neq 0$ are still valid in the symmetric case. The branching families have the analytic form

$$
x=\Phi\left((\sigma-v) \frac{1}{t}, e^{\sigma t / \nu_{0}}\right)
$$

where $\sigma$ is the family parameter and where $\Phi$ is a series of positive powers of $(\sigma-v)^{\frac{1}{2}}$ and of positive and negative powers of $e^{\sigma t / \nu_{0}}$. The series are absolutely convergent (for pure imaginary $\nu$ and $\sigma$ ) when $|\sigma-\nu|$ is sufficiently small. The generating solution is the member of $(1.6)$ for which $\sigma=v$. (The case $v_{0}=3$ is exceptional in that $(\sigma-v)^{\frac{1}{2}}$ may be replaced in $(1.6)$ by $\left.\sigma-v\right)$.

The results are stated to hold in general but exceptional cases may occur when leading coefficients in a certain power series vanish accidentally. In such cases additional branching families may be obtained whose members are unsymmetric. Detailed results for some exceptional cases are stated in sections 5 and 6 .

The results for exceptional cases may be relevant to systems (1.1) which depend analytically on a parameter $\mu$. The coefficients in question are then continuous functions of $\mu$ and so it is possible that they could vanish for a discrete set of values of $\mu$.

The coefficients in question may also vanish because of the presence of 
more than one symmetry property in the Hamiltonian system and the generating solution. In a subsequent paper we shall present the modifications which the theory for such systems requires and we shall give some numerical illustrations.

\section{Outline of procedure}

We shall first of all outline the way in which Cherry obtains the periodic solutions in the case where $\lambda / v$ is rational, the only case in which we are interested here.

Cherry constructs a formal contact transformation to new variables $Z$ which we denote by

$$
x=p\left(Z, e^{\sigma t / \nu_{0}}\right) .
$$

Here $\sigma$ is an arbitrary parameter and the right hand side is a formal power series in the components of the vector $Z$ with coefficients which are Laurent series in $e^{\sigma t j \nu_{0}}$. Under this transformation the system (1.1) assumes a simple normal form

$$
\frac{d Z}{d t}=J L_{z}
$$

The new Hamiltonian $L$ is a formal power series in the $Z_{k}, U_{k}$ with $\sigma-v$ occurring linearly. The variables $Z_{1}$ and $U_{1}$ occur only in the combinations

$$
v=Z_{1} U_{1}, \quad w=Z_{1}^{p_{0}}, \quad w^{\prime}=U_{1}^{p_{0}} .
$$

Equilibrium solutions of the normalized system (2.2) are then found which we denote by

$$
Z=Z^{0}
$$

$Z^{0}$ is given as a function of $\sigma-v$ which vanishes with this argument. When the equilibrium solutions are substituted in (2.1) the families (1.6) of periodic solutions of the original system are obtained. Convergence of the formal series which specify the families (1.6) is then proved by the method of dominant series.

In outline our procedure for specializing Cherry's theory to symmetric systems is as follows: we study the effect of symmetry in the original system (1.1) and in the generating solution (1.5) on the transformation (2.1); on the Hamiltonian $L$; on the equilibrium solutions and then finally on the periodic solutions (1.6) of the original system.

In carrying out this procedure we shall need to consider in some detail the way in which the transformation (2.1) was constructed. It was actually 
composed of four contact transformations and we shall denote the first three of these transformations by

$$
\begin{aligned}
& x=\phi\left(e^{\nu t}\right)+\xi \\
& \xi=\Theta\left(e^{\nu t}\right) \zeta \\
& \zeta=g\left(z, e^{\nu t}\right)
\end{aligned}
$$

Here $x, \xi, \zeta$ and $z$ are vectors; $\phi$ is the generating solution; $\Theta$ is a matrix and $g$ is a vector function of $z$. We shall denote the Hamiltonians of the systems to which (2.6) and (2.7) lead by $G\left(\zeta, e^{\nu t}\right)$ and $K\left(z, e^{\nu t}\right)$ respectively. The transformations are determined in such a way that in the series developments $G$ has its quadratic terms in $\zeta$ normalized while $K$ has all its higher order terms in $z$ normalized.

If in these transformations the constant $v$ is replaced by the arbitrary parameter $\sigma$, the overall transformation becomes $x=f\left(z, e^{\sigma t}\right)$ where

$$
f\left(z, e^{\sigma t}\right)=\phi\left(e^{\sigma t}\right)+\Theta\left(e^{\sigma t}\right) g\left(z, e^{\sigma t}\right) .
$$

When applied to (1.1) this transformation gives a system with Hamiltonian $K\left(\sigma-v, z, e^{\sigma t}\right)$ which is a power series in the arguments

$$
z_{1} u_{1}, \quad z_{1}^{\nu_{0}}\left(e^{\sigma t}\right)^{-\lambda_{0}}, \quad u_{1}^{\nu_{0}}\left(e^{\sigma t}\right)^{\lambda_{0}}, \quad z_{2} \text { and } u_{2}
$$

with $\sigma-v$ occurring linearly.

We shall write the fourth transformation, which leads from Hamiltonian $K$ to Hamiltonian $L$, in component form:

$$
z_{1}=Z_{1}\left(e^{\sigma t}\right)^{\lambda_{0} / \nu_{0}}, \quad z_{2}=Z_{2}, \quad u_{1}=U_{1}\left(e^{\sigma t}\right)^{-\lambda_{0} / \nu_{0}}, \quad u_{2}=U_{2} .
$$

We study in succession the effect of symmetry on each of the above transformations and the Hamiltonians to which they lead. Under the hypothesis $\lambda_{0} \neq 0$ and $\nu_{0} \geqq 3$ we study the effect of symmetry on $\Theta$ and $G$ in section 3 . The bulk of section 4 is then concerned with its effect on $g$ and $K$. The results, stated in theorems 1 and 2, are then pieced together to give the result that the transformation (2.1) has the property

$$
p\left(W Z, e^{-\sigma t / \nu_{0}}\right)=V p\left(Z, e^{\sigma t / \nu_{0}}\right),
$$

where $W$ is a certain involutory matrix, while the Hamiltonian $L$ has the property

$$
L(\sigma-v, W Z)=L(\sigma-v, Z) .
$$

As a consequence of this property of $L$ it is shown in section 5 that the equilibrium solutions (2.4) have in general the property

$$
W Z^{\mathbf{0}}=Z^{\mathbf{0}} \text {. }
$$


The corresponding periodic solutions of the original system $x=p\left(Z^{0}, e^{\sigma t / v_{0}}\right)$, which give the branching families (1.6), therefore have the property

$$
p\left(Z^{0}, e^{\sigma t / \nu_{0}}\right)=V p\left(Z^{0}, e^{-\sigma t / \nu_{0}}\right)
$$

in view of (2.11). Comparing (2.14) with (1.4) we see that the members of the branching families have the same symmetry as the generating solution had.

In sections 6 and 7 we sketch the modifications which are required when $\nu_{0}=2$ and when $\lambda_{0}=0$ respectively.

The discussion is a merely formal one since the convergence theory of $[5]$ is still applicable.

\section{Symmetry and the linear transformation}

If $x=\phi\left(e^{\nu t}\right)+\xi$ is substituted in the differential equations (1.1) and the terms of degree greater than 1 in the components of $\xi$ are then neglected in the series development of the right hand sides, a linear homogeneous system of differential equations with periodic coefficients is obtained

$$
\frac{d \xi}{d t}=J F_{x x} \xi
$$

These equations are called the equations of variation of the system (1.1) with respect to the generating solution (1.5).

The transformation $\xi=\Theta\left(e^{\nu t}\right) \zeta$ is the linear contact transformation with periodic coefficients which brings these equations to a normal form. Such transformations were first given by Cherry but have since been discussed by Moser [8; pp. 87-101]. Siegel [9; $§ 13]$ discusses the equilibrium case, corresponding to $\phi\left(e^{v t}\right)$ being a constant.

In this section we shall assume that $\lambda / v \neq 0$ and $\lambda / v \neq \frac{1}{2}$ and we shall change our notation slightly by writing $\Theta(t)$ in place of $\Theta\left(e^{p t}\right)$. Thus $\Theta(t)$ has period $2 \pi i / v,=T$ say. The properties of $\Theta(t)$ which we shall use in discussing symmetry are listed below. It should be noted that $\Theta(t)$ is by no means uniquely determined by these conditions.

Firstly, the condition that the transformation preserve the Hamiltonian form of the equations may be written in the form (cf. $[9 ;$ p. 11])

$$
\Theta(t)^{\prime} J \Theta(t)=J
$$

Secondly, when the original system and $t$ are real (as we assume), the second and fourth columns $\theta^{(2)}(t)$ and $\theta^{(4)}(t)$ of the matrix $\Theta(t)$ are real. And when $\lambda$ is pure imaginary the first and third columns satisfy

$$
\theta^{(1)}(t)=i \Gamma \overline{\theta^{(3)}(t)}
$$


Here $\Gamma$ is a constant which is equal to 1 or -1 depending on the original system (c.f. Cherry's "case A" and "case B" [5; p. 157]).

Thirdly, $\theta^{(4)}(t)$ is a constant multiple of the vector $d / d t \phi\left(e^{\nu t}\right)$, which is a solution of the equations of variation (c.f. $[10 ; \S 87]$ ).

Finally the normal form to which the transformation brings the equations of variation $(3.1)$ is

$$
\frac{d \zeta}{d t}=J G_{\zeta}^{(2)}
$$

where

$$
G^{(2)}(\zeta)=\lambda \zeta_{1} \omega_{1}-\frac{1}{2} a \zeta_{2}^{2}
$$

When the transformation is applied to the original non-linear system we get a system with quadratically normalized Hamiltonian

$$
G\left(\zeta, e^{\nu t}\right)=G^{(2)}(\zeta)+\cdots
$$

where the terms not written explicitly have degree greater than 2 in the components of $\zeta$. We can now prove

THEOREM 1. When $F$ and $\phi$ have respectively the symmetry properties (1.2) and (1.4), characterized by a matrix $V$, then $\Theta(t)$ may be chosen so that it has the property

$$
V \Theta(t)=\Theta(-t) W
$$

where $W$ is the involutory matrix

$$
\left[\begin{array}{rrrr}
0 & 0 & -i \Gamma & 0 \\
0 & 1 & 0 & 0 \\
i \Gamma & 0 & 0 & 0 \\
0 & 0 & 0 & -1
\end{array}\right]
$$

The Hamiltonian $G$ then has the property

$$
G\left(\zeta, e^{\nu t}\right)=G\left(W \zeta, e^{-\nu t}\right) .
$$

Proof. From the symmetry of $F$ and $\phi$ it follows immediately that if $X(t)$ is a fundamental system of solutions of the equations of variation (3.1) then so is $V X(-t)$. There exists, therefore, a matrix $C$ which is independent of $t$ such that

$$
X(t) C=V X(-t) .
$$

Now it is easily verified that the normalized equations of variation (3.4) admit the fundamental system of solutions 


$$
\Lambda(t)=\left[\begin{array}{llll}
e^{\lambda t} & 0 & 0 & 0 \\
0 & 1 & 0 & 0 \\
0 & 0 & e^{-\lambda t} & 0 \\
0 & \text { at } & 0 & 1
\end{array}\right]
$$

so that the matrix $\Theta(t) \Lambda(t)$ will be a fundamental system of solutions of the original equations of variation (3.1). We may therefore substitute this matrix into $(3.10)$ in place of $X(t)$. We thus get

$$
\Lambda(t) C \Lambda(-t)^{-1}=\Theta(t)^{-1} V \Theta(-t) \text {. }
$$

But since the right hand side has period $T,=2 \pi i / \nu$, we must have

$$
\Lambda(T) C \Lambda(-T)^{-1}=C
$$

since $\boldsymbol{A}(0)$ is the unit matrix.

Let us suppose for the present that $a \neq 0$. The hypothesis on $\lambda / v$ gives $e^{\lambda T} \neq \pm 1$. Hence from the above equation we find on using the explicit form of $\Lambda(T)$ that $C$ must be a matrix of the form

$$
C=\left[\begin{array}{rrrr}
0 & 0 & \delta & 0 \\
0 & \beta & 0 & 0 \\
\alpha & 0 & 0 & 0 \\
0 & \gamma & 0 & -\beta
\end{array}\right]
$$

Direct calculation now gives

$$
\Lambda(t) C \Lambda(-t)^{-1}=C
$$

and so (3.11) becomes simply

$$
\Theta(t) C=V \Theta(-t) .
$$

Thus we have only to show that there is a $\Theta(t)$, satisfying the previous conditions, for which $C=W$.

Firstly, $\theta^{(4)}(t)$ is a multiple of $d / d t \phi\left(e^{\nu t}\right)$ and so from the symmetry of $\phi\left(e^{\nu t}\right)$ we have $\theta^{(4)}(t)=-V \theta^{(4)}(-t)$. If we substitute this result in (3.12) we find that $\beta=1$.

Secondly, we may replace $\Theta(t)$ by $\Theta(t) A$ where $A$ is the matrix

$$
\left[\begin{array}{llll}
1 & 0 & 0 & 0 \\
0 & 1 & 0 & 0 \\
0 & 0 & 1 & 0 \\
0 & \frac{1}{2} \gamma & 0 & 1
\end{array}\right]
$$

This leaves unchanged the conditions $\Theta(t)$ already satisfies except that $C$ is replaced by $A^{-1} C A$ and in this new $C$ we have the new $\gamma=0$. 
Thirdly, we may replace $\Theta(t)$ by the matrix $\Theta(t) B$ where $B$ is the diagonal matrix

$$
\operatorname{diag}\left(e^{i \mu}, 1, e^{-i \mu}, 1\right)
$$

in which $\mu$ is a real number at present arbitrary. Now at least one of the elements of the vector $\theta^{(3)}(0)$ must be non-zero and we shall suppose for definiteness that the first element, $\theta_{31}(0), \neq 0$. We can now choose $\mu$ so that in the new $\Theta(t)$ the new $\theta_{31}(0)$ is either real or pure imaginary as we please. From (3.3) we then get

$$
\theta_{11}(0)= \pm i \Gamma \theta_{31}(0)
$$

where the sign depends on our choice of $\mu$. On the other hand (3.12) gives

$$
\theta_{11}(0)= \pm \propto \theta_{31}(0) \text { and } \theta_{31}(0)= \pm \delta \theta_{11}(0)
$$

where the sign here depends on the particular form (1.3) of the matrix $V$. Comparing these equations we see that $\mu$ can always be chosen so that $\alpha=i \Gamma$ and $\delta=-i \Gamma$.

Thus we have shown that, when $a \neq 0, \theta(t)$ can be chosen so that $C=W$ in (3.12) and hence $V \Theta(t)=\Theta(-t) W$ as desired. A straightforward modification of the above shows that the result is also valid when $a=0$.

To prove the property of the Hamiltonian $G$ we note that by the wellknown rule for transforming Hamiltonian functions $[10 ; \S 27]$

$$
G\left(\zeta, e^{\nu t}\right)=G^{(2)}(\zeta)+F^{*}\left(\phi\left(e^{\nu t}\right)+\Theta(t) \zeta\right)
$$

where $F^{*}(x)$ denotes the sum of those terms in the series development of $F(x)$ whose degree exceeds 2 . On the right hand side of this equation we now consider each member separately. From (3.5) and (3.8) it follows immediately that

$$
G^{(2)}(W \zeta)=\lambda\left(-i \Gamma \omega_{1}\right)\left(i \Gamma \zeta_{1}\right)-\frac{1}{2} a \zeta_{2}^{2}=G^{(2)}(\zeta) .
$$

As regards the second member we note that by (1.4) and (3.7)

$$
\phi\left(e^{-\nu t}\right)+\Theta(-t) W \zeta=V\left(\phi\left(e^{\nu t}\right)+\Theta(t) \zeta\right) .
$$

But it follows from (1.2) that $F^{*}(V x)=F^{*}(x)$. Hence

$$
F^{*}\left(\phi\left(e^{-p t}\right)+\Theta(-t) W \zeta\right)=F^{*}\left(\phi\left(e^{p t}\right)+\Theta(t) \zeta\right) .
$$

From (3.13), (3.14) and (3.15) the required property $G\left(W \zeta, e^{-\nu t}\right)=G\left(\zeta, e^{p t}\right)$ now follows. 


\section{Symmetry and the non-linear transformation}

We shall need the following

LEMma. Let $\zeta=g\left(z, e^{p t}\right)$ be a contact transformation which brings the system with Hamiltonian $G\left(\zeta, e^{\nu t}\right)$ to one with Hamiltonian $K\left(z, e^{\nu t}\right)$. If $G$ has the property that $G\left(\zeta, e^{\nu t}\right)=G\left(W \zeta, e^{-\nu t}\right)$, then the transformation $\zeta=W^{-1} g\left(W z, e^{-\nu t}\right)$ brings the former system to a system with Hamiltonian $K\left(W z, e^{-\nu t}\right)$.

The lemma may be proved by a straight forward application of the rule $[10 ; \S 27]$ by which Hamiltonian functions are transformed and we omit the details.

We shall now consider the effect of symmetry on the transformation (2.7), $\zeta=g\left(z, e^{\nu t}\right)$. When applied to the system with Hamiltonian (3.6),

$$
G\left(\zeta, e^{p t}\right)=\lambda \zeta_{1} \omega_{1}-\frac{1}{2} a \zeta_{2}^{2}+\cdots,
$$

it gives a system with Hamiltonian

$$
K\left(z, e^{\nu t}\right)=\lambda z_{1} u_{1}-\frac{1}{2} a z_{2}^{2}+\cdots
$$

in which only the following combinations of the $z_{k}, u_{k}$ and $t$ appear

$$
v=z_{1} u_{1}, \quad w=z_{1}^{\nu_{0}}\left(e^{\nu t}\right)^{-\lambda_{0}}, \quad w^{\prime}=u_{1}^{\nu_{0}}\left(e^{\nu t}\right)^{\lambda_{0}}, \quad z_{2} \text { and } u_{2} \text {. }
$$

The original procedure for constructing this transformation was given by Cherry [4]. A more direct procedure is due to Birkhoff $[1 ; \mathrm{pp} .85-88]$. Although Birkhoff treats only the incommensurable case explicitly, his procedure can be extended trivially to deal with the case in which $\lambda / \nu$ is rational. By referring to Birkhoff's work we can easily prove

THEOREM 2. Because of symmetry the transformation $\zeta=g\left(z, e^{t t}\right)$ may be determined so that it has the property

$$
g\left(W z, e^{\nu t}\right)=W g\left(z, e^{-\nu t}\right)
$$

while the corresponding Hamiltonian $K\left(z, e^{\nu t}\right)$ has the property

$$
K\left(z, e^{\nu t}\right)=K\left(W z, e^{-\nu t}\right) .
$$

Proof. The leading idea in Birkhoff's construction is to use an auxiliary function $S$ to define the contact transformation. If $S=\zeta_{1} u_{1}+\zeta_{2} u_{2}+\ldots$ is a formal power series in the $\zeta_{k}, \boldsymbol{u}_{k}$ with coefficients which are Laurent series in $e^{p t}$ then the equations

$$
z_{k}=\frac{\partial S}{\partial u_{k}}, \quad \omega_{k}=\frac{\partial S}{\partial \zeta_{k}} \quad(k=1,2)
$$


are uniquely soluble for the $\zeta_{k}, \omega_{k}$ in terms of the $z_{k}, u_{k}$ and so define a contact transformation.

Now it turns out that the coefficients in $S$ can be successively determined so that the only terms which remain in $K$ have the form $A z_{1}^{\alpha_{1}} u_{1}^{\beta_{1}} z_{2}^{\alpha_{2}}$ $u_{2}^{\beta} a\left(e^{v t}\right)^{c}$ with

$$
\lambda\left(\alpha_{1}-\beta_{1}\right)+\nu c=0
$$

(which means that, when $\lambda / \nu$ is rational, $K$ contains only powers of the variables $(4.1))$. In this way the coefficient of every term $\zeta_{1}^{\alpha_{1}} u_{1}^{\beta_{1}} \zeta_{2}^{\alpha_{1}} u_{2}^{\beta_{2}}\left(e^{\nu t}\right)^{0}$ in $S$ is uniquely determined unless the indices satisfy the above condition.

At this stage we determine the coefficients of the excepted terms also by imposing a condition used by Siegel $[9 ;$ p. 187] that in the expansion of

$$
\sum_{k=1}^{2}\left(z_{k} \omega_{k}-u_{k} \zeta_{k}\right)=z^{\prime} J \zeta
$$

in powers of the $z_{k}, u_{k}$ no powers of the variables (4.1) alone should occur. The resulting transformation is thus determined uniquely and we take it as our transformation $\zeta=g\left(z, e^{\nu t}\right)$.

Let us now consider the related transformation

$$
\zeta=W^{-1} g\left(W z, e^{-\nu t}\right)=z+\cdots
$$

which may be represented by a function $S$ as above.

Firstly, in view of the lemma, and (3.9), it leads to a system with Hamiltonian function $K\left(W z, e^{-\nu t}\right)$ and this function involves only powers of the variables (4.1) since the replacement of $z$ by $W z$ and $t$ by $-t$ merely permutes the variables (4.1).

Secondly the expression corresponding to (4.4) is now

$$
z^{\prime} J W^{-1} g\left(W z, e^{-v t}\right) \text {. }
$$

Since $W^{\prime} J W=-J$ this is equal to

$$
-(W z)^{\prime} J g\left(W z, e^{-\nu t}\right)
$$

which again involves no powers of the variables (4.1) alone.

Thus by uniqueness we get the required property of $g$,

$$
W^{-1} g\left(W z, e^{-\nu t}\right)=g\left(z, e^{\nu t}\right) \text {. }
$$

A further application of the lemma now gives $K\left(z, e^{\nu t}\right)=K\left(W z, e^{-\nu t}\right)$, thereby completing the proof of the theorem.

CoRollaRY 1. Because of symmetry the transformation $(2.8), x=f\left(z, e^{\sigma t}\right)$, has the property

$$
V f\left(z, e^{\sigma t}\right)=f\left(W z, e^{-\sigma t}\right)
$$


and the corresponding Hamiltonian $K\left(\sigma-v, z, e^{\sigma t}\right)$ has the property

$$
K\left(\sigma-v, z, e^{\sigma t}\right)=K\left(\sigma-\nu, W z, e^{-\sigma t}\right) .
$$

Proof. The result for $f$ is an immediate consequence of theorems 1 and 2. The result for $K$ is obtained by noting that

$$
K\left(\sigma-v, z, e^{\sigma t}\right)=F\left(f\left(z, e^{\sigma t}\right)\right)+\sigma M\left(z, e^{\sigma t}\right)
$$

where, by the rule $[10 ; \S 27]$ forltransforming Hamiltonian functions, the remainder function $\sigma M\left(z, e^{\sigma t}\right)$ depends on $\sigma$ only as indicated by the notation. From (1.2) and (4.5) we see that

$$
F\left(f\left(W z, e^{-\sigma t}\right)\right)=F\left(f\left(z, e^{\sigma t}\right)\right) .
$$

On the other hand theorem 2 gives

$$
K\left(0, W z, e^{-\nu t}\right)=K\left(0, z, e^{\nu t}\right) .
$$

Substituting these two results in (4.7) with $\sigma=\nu$ we find that

$$
M\left(W z, e^{-\nu t}\right)=M\left(z, e^{\nu t}\right) .
$$

But since this holds identically in $t$ we may replace $v$ by $\sigma$ to get

$$
M\left(W z, e^{-\sigma t}\right)=M\left(z, e^{\sigma t}\right) .
$$

From (4.7), (4.8) and (4.9) we see immediately that $K\left(\sigma-v, z, e^{\sigma t}\right)=$ $K\left(\sigma-v, W z, e^{-\sigma t}\right)$ as required.

COROLlaRY 2. Because of symmetry the transformation (2.1), $x=$ $p\left(Z, e^{\sigma t / \nu_{0}}\right)$, has the property

$$
V p\left(Z, e^{\sigma t / \nu_{0}}\right)=p\left(W Z, e^{-\sigma t / \nu_{0}}\right)
$$

and the corresponding Hamiltonian $L$ has the property

$$
L(\sigma-v, Z)=L(\sigma-v, W Z) .
$$

ProOF. Let us write the transformation (2.10) in vector notation as

$$
z=\chi\left(Z, e^{\sigma t / \nu_{0}}\right) \text {. }
$$

Then from (2.1) and the explicit form (3.8) of the matrix $W$ we see that

$$
\chi\left(W Z, e^{-\sigma t / \nu_{0}}\right)=W \chi\left(Z, e^{\sigma t / \nu_{0}}\right)
$$

since the vectors on either side of this equation each have components

$$
-i \Gamma U_{1}\left(e^{\sigma t}\right)^{-\lambda_{0} / \nu_{0}}, \quad Z_{2}, \quad i \Gamma Z_{1}\left(e^{\sigma t}\right)^{\lambda_{0} / \nu_{0}}, \quad-U_{2} .
$$

Now $p$ may be expressed in terms of $f$ by the equation

$$
p\left(Z, e^{\sigma t / \nu_{0}}\right)=f\left(\chi\left(Z, e^{\sigma t / v_{0}}\right), e^{\sigma t}\right) .
$$


Thus

$$
\begin{aligned}
V p\left(Z, e^{\sigma t / \nu_{0}}\right) & =f\left(W \chi\left(Z, e^{\sigma t / \nu_{0}}\right), e^{-\sigma t}\right) \text { by }(4.5) \\
& =f\left(\chi\left(W Z, e^{-\sigma t / \nu_{0}}\right), e^{-\sigma t}\right) \text { by }(4.12) \\
& =p\left(W Z, e^{-\sigma t / \nu_{0}}\right) .
\end{aligned}
$$

Thus (4.10) is proved.

The property of $L$ now follows from the expression for $L$ in terms of $K$ given in $[5 ;$ p. 163] in a straightforward way and we omit the details.

\section{Solution of the normalized system}

We shall now consider the effect of symmetry on the equilibrium solutions $Z=Z^{0}$ of the normalized Hamiltonian system (2.2) which lead to the branching families.

The procedure by which these solutions are obtained [5; pp. 163-175] may be summarized as follows. In place of the variables

$$
v=Z_{1} U_{1}, \quad w=Z_{1}^{y_{0}}, \quad w^{\prime}=U_{1}^{\nu_{0}}
$$

new variables $q, s, s^{\prime}$ are introduced by putting

$$
q=i \Gamma v, \quad s=w+(i \Gamma)^{v_{0}} w^{\prime}, \quad s^{\prime}=i\left(w-(i \Gamma)^{v_{0}} w^{\prime}\right)
$$

where $\Gamma= \pm 1$, as in section 3. In place of the Hamiltonian $L$ a function $H$, which is a real valued function of its arguments, is introduced by putting

$$
H\left(i(\sigma-v), q, s, s^{\prime}, Z_{2}, U_{2}\right)=L(Z, \sigma-v) .
$$

The series expansion of $H$, which we shall require later, is of the form

$$
\begin{aligned}
H= & i(\sigma-v)\left(\alpha_{1} Z_{2}+\alpha_{2} q+\alpha_{3} s+\alpha_{4} s^{\prime}+\cdots\right) \\
& -\frac{1}{2} a Z_{2}^{2}+\beta_{1} Z_{2} q+\beta_{3} s+\beta_{4} s^{\prime}+\frac{1}{2} \beta_{5} q^{2} \\
& +\beta_{8} q s+\beta_{7} q s^{\prime}+\beta_{8} Z_{2} s+\beta_{9} Z_{2} s^{\prime} \\
& +\frac{1}{2} \beta_{10} s^{2}+\frac{1}{2} \beta_{11} s^{\prime 2}+\cdots
\end{aligned}
$$

where the $\alpha$ 's and $\beta$ 's are real constants. We have not given any terms in $U_{2}$ as these will not be required in the sequel. The following equations are then solved for $q, s, s^{\prime}, Z_{2}, U_{2}$ as functions of $i(\sigma-v)$ which vanish therewith:

$$
\begin{aligned}
& s^{2}+s^{2}-4 q^{\nu_{0}}=0 \\
& s^{\prime} \frac{\partial H}{\partial s}-s \frac{\partial H}{\partial s^{\prime}}=0
\end{aligned}
$$




$$
\begin{aligned}
& s \frac{\partial H}{\partial q}+2 v_{0} q^{\nu_{0}-1} \frac{\partial H}{\partial s}=0 \\
& (s \neq 0) \\
& \text { or } \quad s^{\prime} \frac{\partial H}{\partial q}+2 v_{0} q^{v_{0}-1} \frac{\partial H}{\partial s^{\prime}}=0 \\
& \left(s^{\prime} \neq 0\right) \\
& \frac{\partial H}{\partial Z_{2}}=0 \\
& U_{2}=0 \text {. }
\end{aligned}
$$

From the values of $q, s, s^{\prime}$ obtained in this way, the corresponding values of $v, w, w^{\prime}$ are determined by using (5.2) and the value of $Z_{1}, U_{1}$ are then obtained from (5.1). The values of the $Z_{k}, U_{k}$ so determined are the required equilibrium solutions of (2.2). When $q$ is positive and $s, s^{\prime}$ are real, the corresponding solutions of the original system (1.1) can be shown to be real.

LEMma. Because of symmetry, $H$ has the property

$$
H\left(i(\sigma-v), q, s, s^{\prime}, Z_{2}, U_{2}\right)=H\left(i(\sigma-v), q,(-1)^{\nu_{0}} s,(-1)^{\nu_{0}+1} s^{\prime}, Z_{2},-U_{2}\right)
$$

Proof. From the explicit form (3.8) of the matrix $W$ we see that the substitution

$$
Z \rightarrow W Z
$$

may be written componentwise as

$$
Z_{1} \rightarrow-i \Gamma U_{1}, \quad Z_{2} \rightarrow Z_{2}, \quad U_{1} \rightarrow i \Gamma Z_{1}, \quad U_{2} \rightarrow-U_{2} .
$$

From (5.1) and (5.2) we see that this is equivalent to the substitution

$$
q \rightarrow q, \quad s \rightarrow(-1)^{\nu_{0}} s, \quad s^{\prime} \rightarrow(-1)^{\nu_{0}+1} s^{\prime}, \quad Z_{2} \rightarrow Z_{2}, \quad U_{2} \rightarrow-U_{2} .
$$

From the definition (5.3) of $H$ we now see that the required property of $H$ is equivalent to the property $L(\sigma-v, Z)=L(\sigma-v, W Z)$ proved already.

We can now prove that the equilibrium solutions have the property (2.13) which ensures that the corresponding periodic solutions of the original system are symmetric.

THEOREM 3. Because of symmetry the equilibrium solutions $Z=Z^{0}$ of (2.2) have in general the property

$$
W Z^{0}=Z^{0}
$$

Proof. Suppose first that $\nu_{0}$ is an odd integer. Then by the lemma $H$ becomes an even function of $s$ when we put $U_{2}=0$ (as (5.5.5) requires). Thus (5.5.2) is explicitly

$$
s^{\prime} s\left(\beta_{10}+\cdots\right)-s\left(\beta_{4}+\cdots\right)=0 .
$$


If $\beta_{4} \neq 0$, which we assume to be in general the case (see below), the only solution which vanishes with $\sigma-\nu$ is

$$
s=0 .
$$

From (5.5.1) and (5.5.3) we now get successively

$$
s^{\prime}= \pm 2 q^{\mathbf{y}_{0} / 2} \text { and } \frac{\partial H}{\partial q} \pm \nu_{0} q^{\nu_{0} / 2-1} \frac{\partial H}{\partial s}=0 .
$$

The second of these equations and (5.5.4) now give $q$ and $Z_{2}$ in terms of $i(\sigma-v)$. When $v_{0}>4$ (the only case for which we give details) these equations have the form

$$
\begin{aligned}
& \alpha_{2} i(\sigma-\nu)+\beta_{1} Z_{2}+\beta_{5} q+\cdots=0 \\
& \alpha_{1} i(\sigma-v)-a Z_{2}+\beta_{1} q+\cdots=0 .
\end{aligned}
$$

If $a \beta_{6}+\beta_{1}^{2} \neq 0$, which is so in general, these equations have the unique solution

$$
\begin{aligned}
q & =-\frac{a \alpha_{2}+\alpha_{1} \beta_{1}}{a \beta_{5}+\beta_{1}^{2}} i(\sigma-v)+\cdots \\
Z_{2} & =\frac{\alpha_{1} \beta_{5}-\alpha_{2} \beta_{1}}{a \beta_{5}+\beta_{1}^{2}} i(\sigma-v)+\cdots
\end{aligned}
$$

From (5.2) and (5.1) we now get in succession

$$
-(i \Gamma)^{\nu_{0}} w^{\prime}=w, \quad w=-i s^{\prime} / 2, \quad Z_{1}^{\nu_{0}}=\mp i q^{\nu_{0} / 2}
$$

and hence $Z_{1}=(\mp i)^{1 / v_{0}} q^{\frac{1}{2}}, U_{1}=-i \Gamma(\mp i)^{-1 / v_{0}} q^{\frac{1}{2}}$. The various determinations of the $v_{0}$-th roots in these expressions for $Z_{1}$ and $U_{1}$ lead to the same periodic solutions of the original system $[5 ; p .167]$. Thus we have only two essentially different families in the above results, which correspond to Families II and III. If we now adopt $\pm i$ as our determination of $i^{1 / v_{0}}-$ which is permissible since $\nu_{0}$ is odd - we get for the two families

$$
Z_{1}= \pm i q^{\frac{1}{2}}, \quad U_{1}=\mp \Gamma q^{\frac{1}{2}} .
$$

In either case let $Z_{k}^{0}, U_{k}^{0}$ denote the values of the $Z_{k}, U_{k}$ so obtained. Then from (5.6) and (5.5.5) we get

$$
Z_{1}^{0}=-i \Gamma U_{1}^{0} \text { and } U_{2}^{0}=0 .
$$

Now from the explicit form (3.8) of the matrix $W$ we see that the vector $W Z^{0}$ has components

$$
-i \Gamma U_{1}^{0}, \quad Z_{2}^{0}, \quad i \Gamma Z_{1}^{0}, \quad-U_{2}^{0} .
$$

But by (5.7) these are equal to the respective components $Z_{1}^{0}, Z_{2}^{0}, U_{1}^{0}, U_{2}^{0}$ of the vector $Z^{0}$. Thus $W Z^{0}=Z^{0}$ as required. 
Suppose now that $\nu_{0}$ is an even integer so that $H$ becomes an even function of $s^{\prime}$ on putting $U_{2}=0$. If we proceed as above we find two families of equilibrium solutions and for one of these families we find

$$
Z_{1}=i q^{\frac{1}{2}}, \quad U_{1}=-\Gamma q^{\frac{1}{2}} .
$$

We can then verify the property $W Z^{0}=Z^{0}$ for the family in exactly the same way as above.

To show that the other family leads to symmetric periodic solutions of the original system it is necessary to change the origin of $t$ by $\pi i / \sigma$, however, and we shall not give the details here. (The need for changing the origin of $t$ has a simple geometric interpretation. Let $A$ and $B$ denote the two points at which the orbit of the generating solution crosses the axis of symmetry orthogonally. Then, when $\nu_{0}$ is even, the orbits in one branching family have both of their orthogonal crossings near $A$, while those of the other branching family have both of their orthogonal crossings near $B$.)

When $v_{0}>3$ the branching families are real on one side of the generating solution and complex on the other since $q$ changes sign with $i(\sigma-v)$ provided $a \alpha_{2}+\alpha_{1} \beta_{1} \neq 0$.

When $v_{0}=3$ trivial modifications of the above show that there is only one branching family in general, but it is real on both sides of the generating solution.

Non-vanishing of the coefficients. In proving theorem 3 we have assumed in the case where $v_{0}$ is odd that

$$
\beta_{4} \neq 0, \quad \alpha \beta_{5}^{2}+\beta_{1}^{2} \neq 0 \text { and } a \alpha_{2}+\alpha_{1} \beta_{1} \neq 0 .
$$

Similar assumptions are used in the case $v_{0}$ even. By appropriately modifying examples given by Cherry we can construct symmetric systems in which these conditions are satisfied. Arguments can then be given to show that these conditions are satisfied in general: c.f. [5], footnote to p. 167. Cases in which the above constants vanish are exceptional.

Results for exceptional cases. The important condition in regard to symmetry is that on $\beta_{4}$. It ensures that the variable $s$ vanishes identically and this in turn ensures that the corresponding periodic solutions of the original system are symmetric. The other conditions merely affect the number of families obtained and their reality.

If $\beta_{4}$ vanishes, however, then there will be a solution of (5.5.2) besides the one $s=0$. This leads in general to a pair of branching families, Families $I V_{\mathbf{a}}$ and $I V_{\mathbf{b}}$. Their members are unsymmetric - as can be seen from specific examples - and Family $I V_{b}$ is composed of the reflections of the members of Family $I V_{a}$ in the axis of symmetry. It turns out that members of these two families are in general complex for all real values of the period, $2 \pi i v_{0} / \sigma$. Families I, II and III exist as before, in general, and have symmetric members. 


\section{The case $\lambda_{0} / \nu_{0}=\frac{1}{2}$}

In this case the previous discussion requires modifications which stem from the fact that for the normal form of the quadratic terms of the Hamiltonian we now have

$$
G^{(2)}\left(\zeta, e^{\nu t}\right)=\lambda \zeta_{1} \omega_{1}-\frac{1}{2} a \zeta_{2}^{2}+b \zeta_{1}^{2} e^{-\nu t}
$$

By making a few minor changes in sections 3 and 4 we can show that theorems 1 and 2 remain valid (regardless of whether $a$ or $b$ vanishes) provided that the matrix $W$ is now given by $W=Q\left(e^{\nu t}\right)$ where

$$
Q\left(e^{\nu t}\right)=\operatorname{diag}\left(-e^{-\nu t}, 1, e^{\nu t},-1\right) .
$$

(The present case has an interesting peculiarity: previously the origin of $t$ could be chosen at either of the two orthogonal crossings of the generating solution, but in the present case the result just stated for $W$ is valid for only one of these choices.)

It follows that in corollary 1 (section 4) we must now take $W=Q\left(e^{\sigma t}\right)$ while in both corollary 2 (section 4) and theorem 3 we must take $W=Q(1)$, which is independent of $t$.

The normalized Hamiltonian $L$ is in the present case a power series in the $Z_{k}, U_{k}$ and $i(\sigma-v)$ with real coefficients. It involves $Z_{1}$ and $U_{1}$ only in the combinations

$$
v=Z_{1} U_{1}, \quad w=Z_{1}^{2}, \quad w^{\prime}=U_{1}^{2} .
$$

From corollary 2, as modified above, we deduce that $L$ is an even function of $Z_{1}$ and $U_{2}$ jointly. In terms of the arguments (6.1) this means that $L$ becomes an even function of $v$ when we put $U_{2}=0$. But since $v^{2}=r e w \omega^{\prime}$ this means that $v$ can be omitted as an argument of $L$. The conditions which the $Z_{k}, U_{k}$ must satisfy $[5 ;$ p. 166] therefore simplify to

$$
Z_{1} \frac{\partial L}{\partial w}=0, \quad U_{1} \frac{\partial L}{\partial w^{\prime}}=0, \quad \frac{\partial L}{\partial Z_{2}}=0, \quad U_{2}=0 .
$$

If we put $U_{2}=0$ we have an expression of the form

$$
\begin{aligned}
I= & (\sigma-v)\left(c_{2} Z_{2}+c_{8} w+c_{9} w^{\prime}+\cdots\right) \\
& -\frac{1}{2} a Z_{2}^{2}+b w+c_{13} w Z_{2}+c_{14} w^{\prime} Z_{2} \\
& +\frac{1}{2} c_{17} w^{2}+\frac{1}{2} c_{18} w^{\prime 2}+c_{19} w w w^{\prime}+\cdots
\end{aligned}
$$

and the above equations are respectively

$$
\begin{aligned}
& Z_{1}\left(c_{8}(\sigma-v)+b+c_{13} Z_{2}+c_{17} w+c_{19} w^{\prime}+\cdots\right)=0 \\
& U_{1}\left(c_{8}(\sigma-v)+c_{14} Z_{2}+c_{18} w+c_{18} w^{\prime}+\cdots\right)=0 \\
& c_{2}(\sigma-v)-a Z_{2}+c_{13} w+c_{14} w^{\prime}+\ldots=0
\end{aligned}
$$


Now in general $b \neq 0$. From (6.2.1) it follows that in this case the only solution which vanishes with $\sigma-\boldsymbol{v}$ has

$$
Z_{1}=0 \text {. }
$$

Assuming that $a \neq 0$ and $c_{14}^{2}+a c_{18} \neq 0$ we find that (6.2) admit the following solutions:

$$
\begin{aligned}
& Z_{1}=0, \quad Z_{2}=a^{-1} c_{2}(\sigma-v)+\cdots, \quad U_{1}=0, \quad U_{2}=0 \\
& Z_{1}=0, \quad Z_{2}=\frac{c_{2} c_{18}-c_{9} c_{14}}{c_{14}^{2}+a c_{18}}(\sigma-v)+\cdots \\
& U_{1}= \pm\left[\left(-\frac{a c_{9}+c_{2} c_{14}}{c_{14}^{2}+a c_{18}}(\sigma-v)\right)^{\frac{1}{2}}+\cdots\right], \quad U_{2}=0 .
\end{aligned}
$$

The ambiguity of sign for $U_{1}$ corresponds to two determinations of the same periodic solution of the original system. Thus (I) and (II) lead back to two families of periodic solutions of the original system, Families I and II. From the conditions $Z_{1}=0, U_{2}=0$ it is easy to verify that theorem 3 holds for the equilibrium solutions (I) and (II). Hence Families I and II are composed of symmetric periodic solutions. Family II is in general real on one side of the generating solution and complex on the other.

Results for exceptional cases. In exceptional cases coefficients which are in general non-zero may vanish. We shall consider the case in which $b=0$. Here $Z_{1}=0$ is not the only solution of $(6.2 .1)$ and we obtain, besides (I) and (II), the following solutions of (6.2):

$$
\begin{aligned}
& Z_{1}= \pm\left[\left(-\frac{a c_{8}+c_{2} c_{13}}{c_{13}^{2}+a c_{17}}(\sigma-v)\right)^{\frac{1}{2}}+\cdots\right] \\
& Z_{2}=\frac{c_{2} c_{17}-c_{8} c_{13}}{c_{13}^{2}+a c_{17}}(\sigma-v)+\cdots \\
& U_{1}=0, \quad U_{2}=0 \\
& Z_{1}= \pm\left[\left(\left(\Delta_{1} / \Delta\right)(\sigma-v)\right)^{\frac{1}{2}}+\cdots\right], \quad Z_{2}=\left(\Delta_{3} / \Delta\right)(\sigma-v)+\cdots \\
& U_{1}= \pm\left[\left(\left(\Delta_{2} / \Delta\right)(\sigma-v)\right)^{\frac{1}{2}}+\cdots\right], \quad U_{2}=0
\end{aligned}
$$

where the $\Delta$ 's are third order determinants involving the $c$ 's. We assume that $c_{13}^{2}+a c_{17} \neq 0$ and $\Delta \neq 0$ in (III) and (IV) respectively. For real periodic solutions of the original system the $Z_{k}, U_{k}$ and $i(\sigma-v)$ must be real. Stability may be studied as in [5]. Our results are as follows.

In the exceptional case $b=0$ we obtain the following branching families of periodic solutions: Families II, III, IV $V_{\mathbf{a}}$ and IV $_{\mathbf{b}}$. The members of Families II and III are symmetric but those of Families $I_{\mathrm{a}}$ and $I V_{b}$ are unsymmetric. 
Family $I V_{\mathrm{a}}$ is composed of the reflections in the axis of symmetry of the members of Family $I V_{b}$.

Family II is real on one side of the generating solution (corresponding to $\sigma=\nu$ ) and complex on the other and the same is true for Family III. Family $I V_{a}$ (and $I V_{b}$ ) is either real on one side of the generating solution and complex on the other or else complex throughout.

The non-zero characteristic exponents of the members of Family I are pure imaginary on both sides of the generating solution or else complex on both sides. For the real members of the branching families the characteristic exponents may be real or pure imaginary (and here the result for one branching family is independent of that for the others).
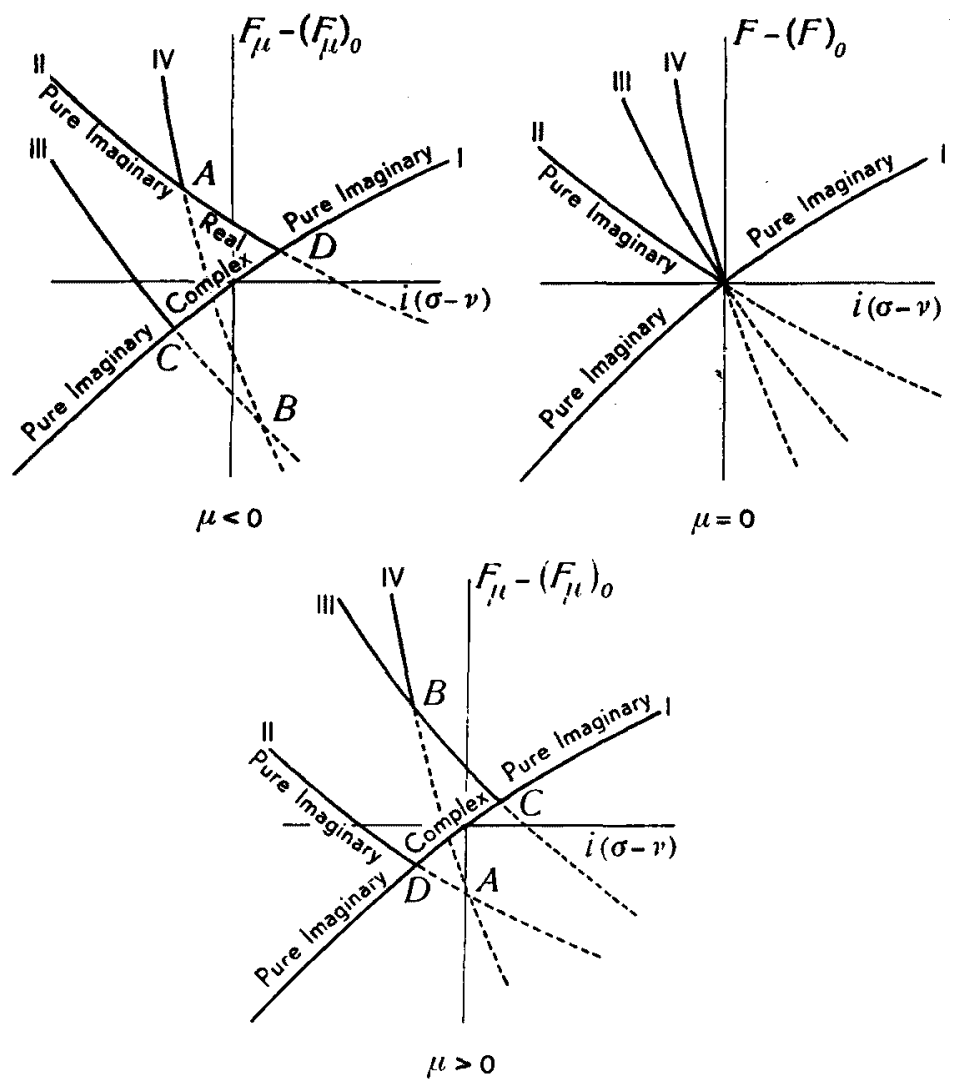

Fig. 1. $v_{0}=2, b=0, a \neq 0, c_{14}^{2}+a c_{18} \neq 0, c_{13}^{2}+a c_{17} \neq 0, a c_{9}+c_{2} c_{16} \neq 0, a c_{8}+c_{2} c_{33} \neq 0$, $\Delta \neq 0, \Delta_{1} \Delta_{2}>0$.

Along each curve is written the nature of the non-zero exponents of the periodic solutions represented by its points. 
Variation of a parameter $\mu$. Suppose now that the original Hamiltonian system (1.1) depends analytically on a parameter $\mu$ and that it is symmetric for each value of $\mu$. Suppose furthermore that for $\mu=0$ the system has a symmetric generating solution with $\lambda / \nu=\frac{1}{2}$ and suppose that it gives the exceptional case $b=0$ as above. As in [5; pp. 210-216] we have studied the periodic solutions near the generating solution when $\mu \neq 0$. The sort of results we have obtained are illustrated in figure 1, which is analogous to those given in [5].

Families $I V_{a}$ and $I V_{b}$ are represented by the same curve. The members of Families I, II and III remain symmetric when $\mu \neq 0$ and those of Families $I_{\mathrm{a}}$ and $I V_{\mathrm{b}}$ remain unsymmetric.

When $\mu \neq 0$ branching occurs at periodic solutions which are represented by the points $A, B, C$ and $D$ in the figure. For $C$ and $D$ the characteristic ratio is $\frac{1}{2}$ and at these points we have instances of the general case, treated earlier in this section. For $A$ and $B$, however, the characteristic ratio is zero and at these points we have instances of a case which is treated later, in section 7.

It can be shown that when for $\mu=0$ the Families IV are complex throughout then the periodic solutions represented by the points $A$ and $B$ are both real for $\mu<0$ (say) and both complex for $\mu>0$. If, on the other hand, the Families IV change from real to complex at the generating solution $\mu=0-$ the situation depicted in figure 1 - then the periodic solutions represented by $A$ and $B$ are real and complex respectively for $\mu<0$ (say) but complex and real respectively for $\mu>0$.

\section{The case $\lambda_{0}=0$}

The normal form of the quadratic terms of the Hamiltonian is now given by

$$
G^{(2)}(\zeta)=a \zeta_{2} \omega_{1}+\frac{1}{2} b \zeta_{1}^{2}+c \zeta_{1} \zeta_{2}+\frac{1}{2} d \zeta_{2}^{2}
$$

By considering the Jordan normal form of the monodromy matrix of the equations of variation of the original system (1.1) we find that in regard to the constants $a, b, c$ and $d$ there are essentially only four cases which can occur:

$$
\begin{aligned}
& a b \neq 0 \\
& a=c=0, \quad b d \neq 0 \\
& a=c=d=0, \quad b \neq 0 \\
& a=b=c=d=0
\end{aligned}
$$

A discussion analogous to sections 3 and 4 now gives the following results. Let $V_{1}=\operatorname{diag}(1,-1,-1,1)$ and let $V_{2}=\operatorname{diag}(1,1,-1,-1)$. 
Then in case (i) theorem 1 and theorem 2 and both corollaries of theorem 2 remain valid if $W$ is replaced by $-V_{1}$. In case (ii), however, we must be prepared to replace $W$ by $V_{1},-V_{1}$ or $V_{2}$ while in cases (iii) and (iv) $W$ must be replaced by $V_{1},-V_{1}, V_{2}$ or $-V_{2}$.

We now give the discussion corresponding to section 5 . The normalized Hamiltonian $L$ is now a power series in the $Z_{k}, U_{k}$ and $i(\sigma-v)$ with real coefficients and we have

$$
\begin{aligned}
L= & (\sigma-v) c_{1} Z_{1}+c_{2} Z_{2}+d_{1} U_{1}+d_{2} U_{2} \\
& +a Z_{2} U_{1}+\frac{1}{2} b Z_{1}^{2}+c Z_{1} Z_{2}+\frac{1}{2} d Z_{2}^{2}+\cdots
\end{aligned}
$$

with at least one of $c_{1}, c_{2}, d_{1}, d_{2}$ non-zero [5;p. 181]. If $c_{2} \neq 0$ we obtain the required equilibrium solutions of the normalized system by putting $U_{2}=0$ and then solving the equations

$$
\frac{\partial L}{\partial Z_{1}}=\frac{\partial L}{\partial Z_{2}}=\frac{\partial L}{\partial U_{1}}=0
$$

for the $Z_{k}, U_{k}$ as functions of $\sigma-\nu$.

In case $(i)$ the solution of equations $(7.1)$ which is given in $[5 ;$ p. 183] is still valid. Family $I$ is the only family of periodic solutions which contains the generating solution and its members are easily shown to be symmetric. (The existence of this case appears to have been overlooked in [7; pp. 73, 74]. An example to illustrate this case is given later.)

Other cases. By analogy with the cases treated in sections 5 and 6 one might expect case (i) to be the general case and cases (ii), (iii) and (iv) to be exceptional. It turns out, however, that among the latter cases there are some which are likely to be of general occurrence for the following reason: if one of these cases occurs for a system (1.1) then it occurs for all neighbouring systems.

Branching families with unsymmetric members are obtained in some of the cases just mentioned.

Variation of $\mu$. We shall exemplify the preceding remarks by considering case (ii) with $W=V_{2}$. We suppose that the original symmetric system (1.1) depends analytically on a parameter $\mu$ and that for $\mu=0$ there is a symmetric generating solution $S_{0}$ of zero characteristic ratio. We suppose further that $S_{0}$ belongs to case (ii) with $W=V_{\mathbf{2}}$. Under these hypotheses we shall now show that for each (sufficiently small) value of $\mu$ there is a periodic solution $S_{\mu}$ of zero characteristic ratio which belongs to the same case as $S_{0}$, namely, case (ii) with $W=V_{2}$.

The Hamiltonian $L$ now involves $\mu$ and the equations (7.1) are to be solved for the $Z_{k}, U_{k}$ as series in $\sigma-v$ and $\mu$ which vanish therewith.

From corollary 2 (section 4 ) as modified above we find that $L$ is an even function of $U_{1}$ and $U_{2}$ jointly and so either $c_{1} \neq 0$ or $c_{2} \neq 0$ by [5; 
p. 181]. After interchanging subscripts if necessary we may suppose that $c_{2} \neq 0$.

Thus with $U_{2}=0$ we have

$$
\begin{aligned}
L= & (\sigma-v)\left(c_{1} Z_{1}+c_{2} Z_{2}+c_{3} U_{1}^{2}+\cdots\right) \\
& +\mu\left(c_{1}^{\prime} Z_{1}+c_{2}^{\prime} Z_{2}+c_{3}^{\prime} U_{1}^{2}+\cdots\right) \\
& +\frac{1}{2} b Z_{1}^{2}+\frac{1}{2} d Z_{2}^{2}+c_{4} Z_{1} U_{1}^{2}+c_{5} Z_{2} U_{1}^{2} \\
& +c_{6} U_{1}^{4}+\cdots
\end{aligned}
$$

The equations (7.1) now become explicitly

$$
\begin{gathered}
c_{1}(\sigma-\nu)+c_{1}^{\prime} \mu+b Z_{1}+c_{4} U_{1}^{2}+\cdots=0 \\
c_{2}(\sigma-\nu)+c_{2}^{\prime} \mu+d Z_{2}+c_{5} U_{1}^{2}+\cdots=0 \\
2 U_{1}\left(c_{3}(\sigma-\nu)+c_{3}^{\prime} \mu+c_{4} Z_{1}+c_{5} Z_{2}+2 c_{6} U_{1}^{2}+\cdots\right)=0
\end{gathered}
$$

These equations may now be solved to give in general the solutions

$$
\begin{aligned}
& Z_{1}=-b^{-1} c_{1}(\sigma-v)-b^{-1} c_{1}^{\prime} \mu-\cdots, \quad U_{1}=0, \\
& Z_{2}=-d^{-1} c_{2}(\sigma-v)-d^{-1} c_{2}^{\prime} \mu-\cdots, \quad U_{2}=0, \\
& Z_{1}=A(\sigma-v)+A^{\prime} \mu+\cdots, \quad U_{1}^{2}=C(\sigma-v)+C^{\prime} \mu+\cdots \\
& Z_{2}=B(\sigma-v)+B^{\prime} \mu+\cdots, \quad U_{2}=0,
\end{aligned}
$$

where $A, B$ and $C$ are constants which depend on the $c$ 's.

From (I) we get a family of symmetric periodic solutions of the original system, Family I, which is real on both sides of the generating solution. But from (II) we get a pair of branching families with unsymmetric members, Families $\mathrm{II}_{\mathrm{a}}$ and $\mathrm{II}_{\mathrm{b}}$, which are real on one side of the generating solution and complex on the other in general. Family $\mathrm{II}_{\mathrm{a}}$ is composed of the reflections in the axis of symmetry of the members of Family $\mathrm{II}_{b}$ and hence these two families are represented by a single curve in figure 2 .
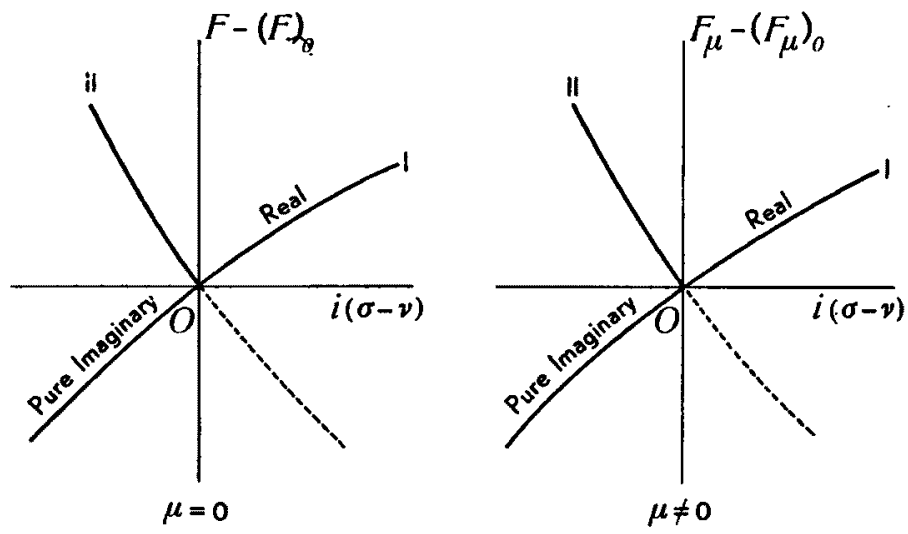

Fig. 2. $\lambda_{0}=0, a=c=0, b d \neq 0, W=V_{2}, C \neq 0, Q \neq 0$ 
Now provided $C \neq 0$ Family I and the Families II have a member in common for each value of $\mu$. It is the member (of either family) for which

$$
\sigma-v=-\frac{C^{\prime}}{C} \mu+\cdots
$$

and it is represented in figure 2 by the origin 0 . This is the periodic solution $S_{\mu}$ whose existence was to be established. It is evident from the figure that the branching at $S_{\mu}$ for $\mu \neq 0$ is of the same sort as that which takes place at $S_{0}$. This suggests that $S_{\mu}$ belongs to the same case as $S_{0}$ and this can be verified by deriving the equations of variation with respect to $S_{\mu}$.

We have calculated the non-zero characteristic exponents $\pm \bar{\lambda}$ of a typical member of Family $I$ as in $[5 ;$ p. 183] and obtained an expression of the form

$$
\tilde{\lambda}^{2}=\left(P(\sigma-v)+P^{\prime} \mu+\cdots\right)\left(Q+R(\sigma-v)+R^{\prime} \mu+\cdots\right)
$$

where $P, Q$ and $R$ are constants depending on the $c$ 's. From this expression we have deduced the results for the non-zero characteristic exponents of the members of the various families in figure 2.

Example of case (i). An example of a symmetric Hamiltonian system for which case (i) occurs can be obtained by specializing the above discussion. We consider the special case in which $Q=0$ in the expression $\tilde{\lambda}^{2}$. In this case $\bar{\lambda}^{2}$ changes sign twice along Family I near the generating solution - once at $S_{\mu}$ and again at a periodic solution $T_{\mu}$ say. We can show by examples that in general $S_{\mu} \neq T_{\mu}$ unless $\mu=0$. Thus we have the situation represented in figure 3 where the point $A$ represents $T_{\mu}$.

$T_{\mu}$ is thus a symmetric periodic solution with all its characteristic exponents zero and it belongs to only one family of periodic solutions. This
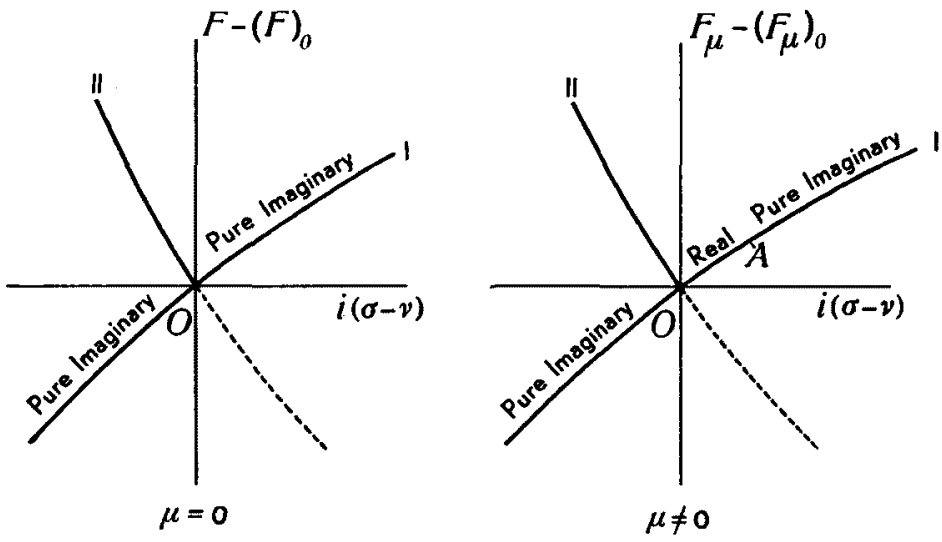

Fig. 3. $\lambda_{0}=0, \quad a=c=0, \quad b d \neq 0, \quad W=V_{2}, \quad C \neq 0, \quad Q=0$ 
suggests that $T_{\mu}$ gives an instance of case (i) and this has in fact been verified by deriving the equations of variation with respect to $T_{\mu}$.

\section{Acknowledgements}

Professor T. M. Cherry suggested the problem to me and most of the results were obtained while I was on a University of Melbourne Research Scholarship.

\section{References}

[1] Birkhoff, G. D., Dynamical Systems, Amer. Math. Soc. (1927).

[2] Birkhoff, G. D., Collected Mathematical Papers, Amer. Math. Soc. (1950), Vol. I and II.

[3] Cherry, T. M., On the transformation of Hamiltonian systems of linear differential equations with constant or periodic coefficients, Proc. Lond. Math. Soc. 26 (1927), $211-230$.

[4] Cherry, T. M. On the solution of Hamiltonian systems of differential equations in the neighbourhood of a singular point, Proc. Lond. Math. Soc. 27 (1927), 151-170.

[5] Cherry, T. M., On periodic solutions of Hamiltonian systems of differential equations, Phil. Trans. Roy. Soc. A, 227 (1928), 137-221.

[6] De Vogelaere, R., Equation de Hill et problème de Störmer, Canad. Jour. Math. 2 (1950), 440-456.

[7] De Vogelaere, $\mathbf{R}$, On the structure of symmetric periodic solutions of conservative systems with applications, Contributions to the Theory of Nonlinear Oscillations, IV, Princeton (1958), 53-84.

[8] Moser, J., New aspects in the theory of stability of Hamiltonian systems, Comm. Pure Appl. Math. 11 (1958) 81-114.

[9] Siegel, C. L., Vorlesungen aber Himmelsmechanik, Springer (1956).

[10] Wintner, A., The Analytical Foundations of Celestial Mechanics, Princeton, (1947).

Australian National University

Canberra, A.C.T. 